\title{
Open Source Hard- and Software: using Arduino boards to keep old hardware running
}

\author{
Helmut Faugel, Volodymyr Bobkov \\ Max-Planck-Institut für Plasmaphysik, EURATOM Association, Garching, Germany
}

\begin{abstract}
The ASDEX Upgrade tokamak went into operation in 1991 with a proposed lifetime of 10 years. Due to major modifications ASDEX Upgrade is still in operation. Infrastructure like data acquisition, workstations, etc. is being modernized, interfaces like RS-232 are vanishing and new interfaces are being introduced. This leads to the necessity to adapt old hardware. Most of the microcontrollers used in the old hardware do not offer any support of the new interfaces and have to be replaced. A simple and efficient way is to replace them with open hardware microcontroller boards like the Arduino. These boards are based on 8-bit RISC microcontrollers and offer a software development environment with a large number of libraries. In this paper the use of Arduino boards for replacing the position unit, the stub tuner interface and its use controlling a direct digital synthesizer (DDS) with phase control capability is shown.
\end{abstract}

Keywords: Data acquisition, Control

\section{Introduction}

The designed lifetime of the ASDEX Upgrade tokamak, which went in operation in 1991, was 10 years. Due to major updates the experiment is still capable to produce valuable results as a part of the ITER step ladder [1] which leads to an experiment program that will last beyond 2016 [2].

The ion cyclotron range of frequency (ICRF) system at ASDEX Upgrade is mainly used for plasma heating, but also other tasks like rf-glow discharges for vessel cleaning can be performed. The main parts of the system are four rf-generators, transmission lines with the matching system and four two straps antennas in the vessel. Subsystems for cooling, pressurized air, vacuum and a data acquisition are needed for running the ICRF experiments at ASDEX Upgrade. The rf-matching of the antennas, which is essential to deliver the power from the rf-generators to the antennas, is done using a double stub tuner system. The positions of these stub tuners are calculated based the measured ratio and phase between forward and reflected power near the matching system of the previous shot.

As two pairs of two rf-generators are connected to a 3-dB hybrid system to minimize the reflected power at the rf-generators caused by fast transient events like ELMs, an active phase control of each generator pair is needed. Currently this is realized with an analog controller.

\section{Interfacing ICRF subsystems}

The operation of an ICRF system in the IPP started in the early 1980s at the ASDEX tokamak. At that time a number of decisions about electric and electronic interfaces for subsystems like rf-generators, transmission lines, vacuum system, etc had to be made. As ASDEX ended operation in 1990 and the ICRF on ASDEX
Upgrade started operation in 1992, much of the control and data acquisition was transferred and adopted to the new ASDEX Upgrade ICRF facility, which operates four rf-generators and four antennas.

One thing which moved en bloc was the CAMAC data acquisition system, which is now outdated and will be replaced soon by the SIO system [3]. Other parts like the stub tuner interface were adapted to the increased number of generators and antennas. Few systems were new developments like the local timer, which triggers the rf-generator during an ASDEX Upgrade shot.

A number of these interface solutions incorporated microprocessors which were programmed in Assembler. The program code is stored in EPROM's (erasable permanent read only memory), which have nowadays exceeded their guaranteed data retention time.

\section{Keeping aged hardware working}

Today the in-house built interfaces are outdated because modern computer offer new interfaces and also a number of electronic or parts used in key components like the stub tuners are no longer available. This ranges from printed circuit board transformers and rectifiers for power supply to connectors for the optical fibers for communication and the rotary resolver for position readout. While it is normally not very difficult fitting in a replacement for transformers and rectifiers, the discontinuing of the connectors for the optical fibers is a major problem. In order to have a consistent system, which allows the exchange of all components of the stub tuner system, all connectors on the optical fibers and the send and receive modules have to be replaced. As the send and receive interfaces on the stub tuner control unit and the stub tuner use RS-422 signaling, some modifications on the printed circuit board have also to be made for this replacement, as fiber optic electronic parts today are using TTL levels. 


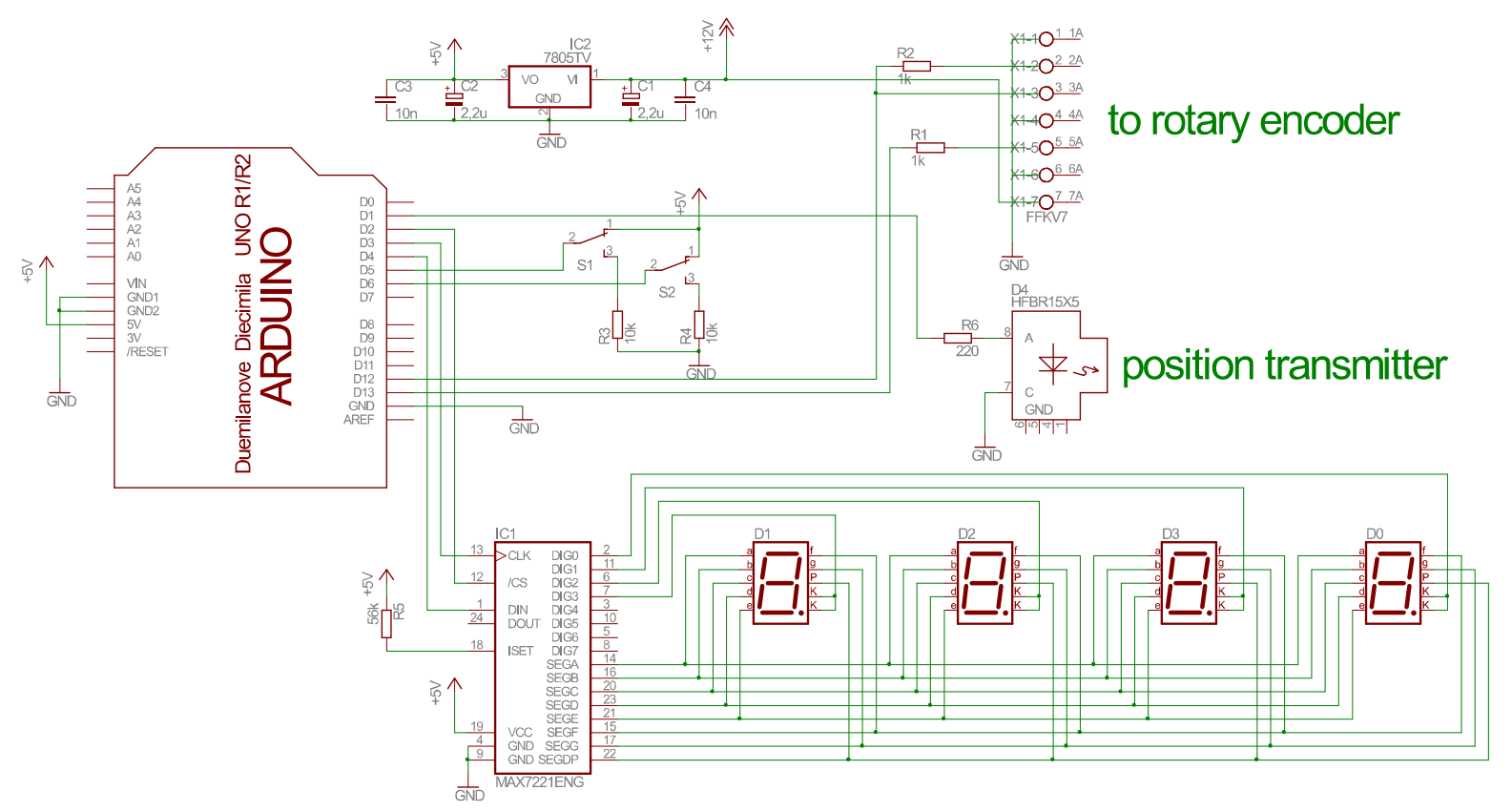

Fig.1 Schematic of the stub tuner position encoder realized with an Arduino Duemilanove board which features 14 digital I/Os and 6 analog inputs. The "position shield" features a four digit 7-segment LED display showing the actual position of the stub tuner, two miniature switches for programming the reference position, the interface to the multi turn rotary encoder which is mechanically connected via drive belt with the spindle that moves the short circuit of the stub tuner. The position which is calculated based on the readout of the rotary encoder and transmitted as a serial data stream via a fiber optical link. The program code written in $\mathrm{C} / \mathrm{C}++\mathrm{using}$ libraries for the LED-display driver IC and EEPROM usage contains less than 200 lines and needs about $6 \mathrm{kB}$ of the $32 \mathrm{kB}$ EEPROM program memory of the microcontroller.

Furthermore a number of modifications on other systems had to be made, like a switch-over from a PC based data acquisition system for monitoring the vacuum feed troughs to a system that is now PLC-based (programmable logic controller). To allow preventive maintenance of the vacuum system, the temperature of the cold heads is now monitored, indicating issues in the system.

\section{Open source hardware}

In contrast to free or open source software, which is already widely used in fusion community, ranging from data mining to publishing, open hardware is quite new. One of the open source hardware projects that quickly became popular is Arduino. It was created in 2005 at the Interaction Design Institute Ivrea (Italy) as a system that allowed students to develop interactive designs. The 16 $\mathrm{MHz}$ 8-bit RISC microcontroller the Arduino uses offers a computing power of about 300.000 lines of program code per second and sufficient in- and outputs for many applications. In addition to the hardware an integrated development environment (IDE) for host computers running the operation systems Linux, MacOS and Windows is available. The programming language is $\mathrm{C} / \mathrm{C}++$ and a number of libraries makes standard applications like printing on an alphanumeric LCD or using serial communication simple. The board can programmed using an USB-interface, the program is stored in the internal EEPROM of the microcontroller. A standardized pin out of the different boards allows connecting a variety of add-on modules called shields. One of the most useful shields is the Ethernet shield, which allows the microcontroller to exchanges data with computers in the local network. An extensive documentation about the hard- and software can be found in [5].

\section{Replacing obsolete parts in the stub tuner}

The position readout system of the stub tuner consists of a 300:1 reduction gear, a 13-bit Gray-code absolute single turn rotary encoder with parallel data outputs and an Intel 8748 microcontroller which converts the readout of the encoder into the position which is transmitted to the stub tuner controller. In order to get rid of a mechanical hysteresis of the position readout the reduction gear is spring loaded. This makes maintenance and service tasks challenging. The rotary encoder used in the stub tuner is also no longer available.

A replacement for the reduction gear and the encoder utilizes a 24-bit multi-turn encoder with a serial interface that is connected to an Ardunio Duemilanuve board. This board offers 6 analog inputs, 14 digital in/outputs (I/O) and a serial interface. Two digital $\mathrm{I} / \mathrm{O}$ are used to read the information from the multi-turn encoder, another three digital I/Os are used to display the current position of the stub tuner, which makes service easier [Fig. 1]. Instead of the complicated procedure needed to readjust the proper position, two switches are used to program the reference position. The reference position is permanently stored in the internal EEPROM of the microcontroller. 


\section{A new stub tuner interface}

The ICRF system uses a shot by shot matching procedure to maintain maximum power coupled to the plasma. The new positions for the stub tuners are calculated from the data acquired at the previous shot. As the data acquisition was en bloc moved from ASDEX to the ASDEX Upgrade ICRF system, the old CAMAC based parallel input/output interface (PIO) was upgraded to control eight stub tuners. Due to the pending changes of the ICRF system [4] of installing two 3-strap antennas in ASDEX Upgrade, two additional transmission lines and matching systems are needed. Therefore a new stub tuner control systems has to be developed, which uses an Ethernet interface. An Arduino board is equipped with an Ethernet shield, and the stub positions are received from the data acquisition computer. This data is processed in the Arduino board and via serial interface transmitted to six Arduino Mega2560 boards. Each of these boards has four serial interfaces, where one is used to communicate with the master Arduno. Two other serial interfaces are used to communicate with two stub tuner control units [Fig. 2].

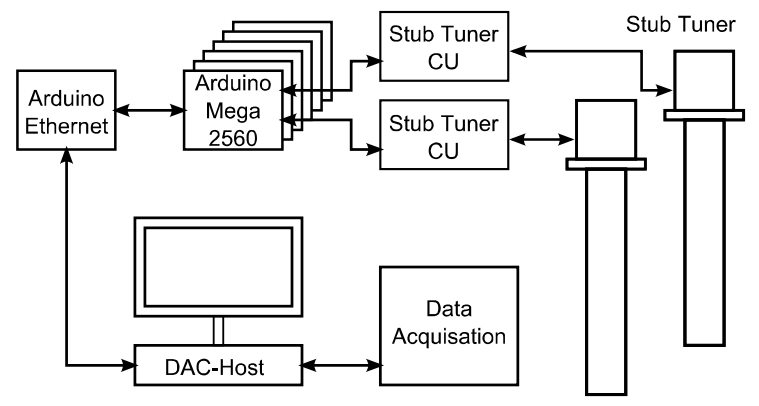

Fig. 2 The new stub tuner control system is using different kinds of Arduino boards. The Arduino Ethernet board is used to communicate with the data acquisition computer for reading and writing (setting) stub tuner positions. The board is also connected with six Arduino Mega2560 boards; each of the boards is connected to two stub tuner control units. The system can be expanded by ether using the forth serial link of the Arduino Mega2560 board or by adding additional boards.

\section{Digitizing the frequency and phase control}

The current configuration of the ICRF system consists of two pairs of generators which are phase controlled to add the power in a 3-dB hybrid. This power is then splitted by another 3-dB hybrid and the power is transmitted to two antennas. To add up the power at the input of the 3-dB combiner hybrid a phase shift of $90^{\circ}$ at its inputs is needed. Due to amplitude-phase modulation effects [6], which are present in high power amplifiers, an active control of the phase between two generators is necessary. The analog phase control system, which is currently in operation, is a complex electronic circuit containing about 15 analog and 10 digital ICs. The phase is shifted on the $10 \mathrm{MHz}$ reference signal path of the signal generators and phase shift is multiplied by the fraction of the output signal frequency divided by 10 MHz. This causes a variable loop amplification of the phase locked loop (PLL) that makes the phase signal prone to ringing during the power ramp up of the generators. Some experiments using a four-channel direct digital synthesizer have been already performed $[7,8]$. The first results using a DDS also showed that the simply setting an output to a new phase value results in a broadband signal which is reflected by the matching system causing a trip of the generators. As a double stub tuner matching system has a limited bandwidth a band pass filter was used for the simulated transient response of $180^{\circ}$ phase jump [Fig. 3].

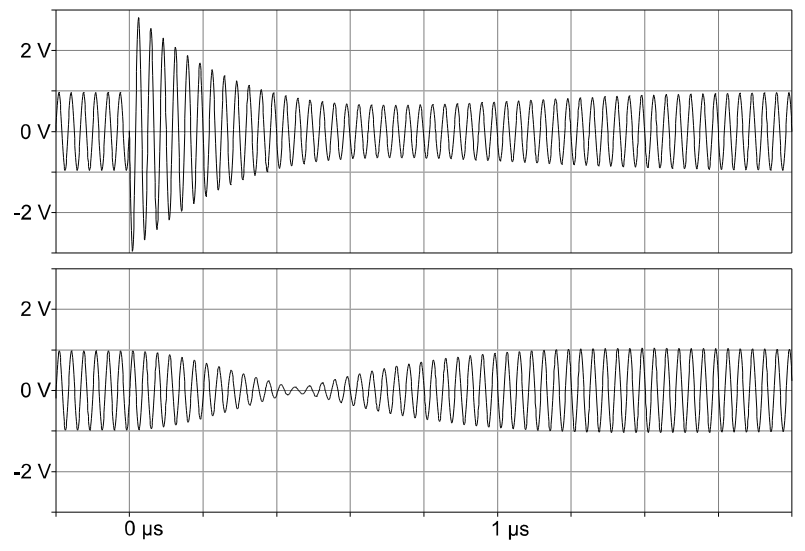

Fig. 3 The two traces are showing the transient behavior of 30 $\mathrm{MHz}$ band pass filter with $1 \mathrm{MHz}$ bandwidth when a $30 \mathrm{MHz}$ input signal makes a phase jump of $180^{\circ}$. The upper trace shows the voltage at the input, the increase of the voltage indicates total reflection of the signal due to its temporary high bandwidth. As a reaction to the total reflection at the input the voltage of the output, lower trace, drops. The bandwidth of the ICRF matching system at ASDEX Upgrade during plasma operation would be about one magnitude smaller and the transient reflection would shut of the ICRF generators.

Because of this behavior, a different approach for the phase control using the DDS is needed. Instead of setting the phase to new values by updating the phase shift registers in the DDS, the phase can be shifted by switching between two different frequencies which are within the bandwidth of the matching system, preventing reflection at the matching system, as the phase is the integral of the frequency difference over a certain period of time [Fig. 5].

To implement a digital phase controller we decided to choose is the DDS IC AD9959 from Analog Devices which has four outputs and a number of different operation modes. The IC offers a serial peripheral interface (SPI) for programming all settings like operation modes, amplitude, frequency and phase. Four digital inputs can be used to modulate the outputs.

To control the DDS IC an Arduino Mega2560 is used as the phase control unit needs a large number of digital I/Os for interfacing the DDS IC, a four line LED display, local control etc. An Ethernet shield is added to communicate with the host computer [Fig. 4]. 


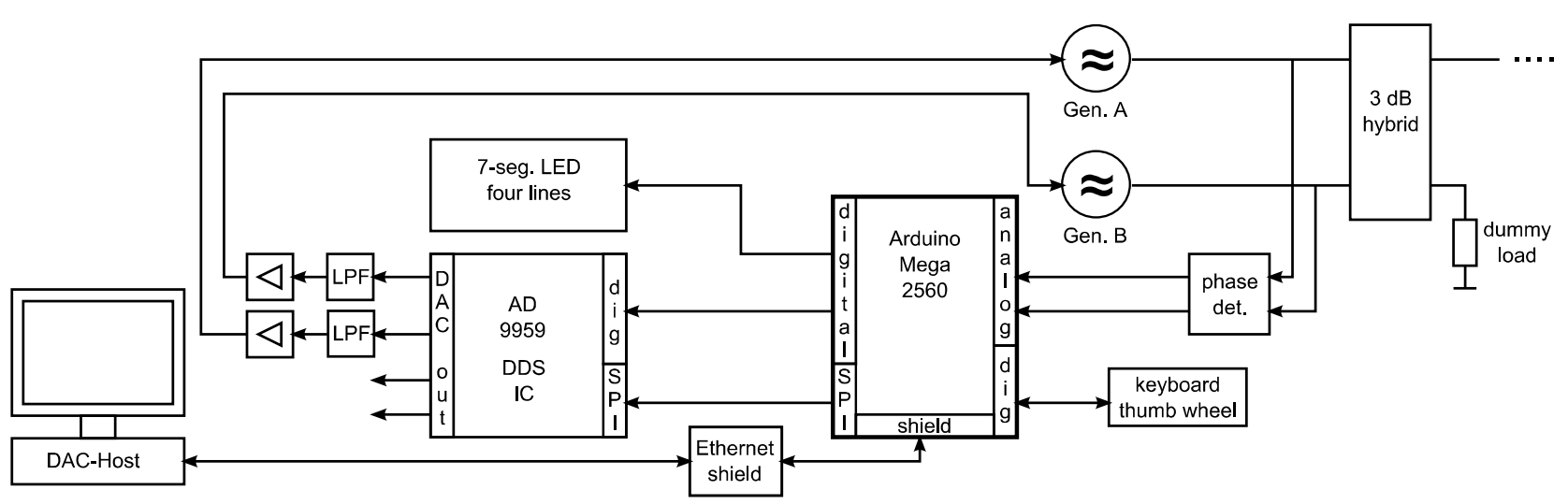

Fig. 4 Digital frequency and phase control shown for two rf-generators. The phase shift between the two generators is measured by a phase detector; the voltages are digitized by the analog inputs of the microcontroller board. The program calculats the phase shift and the deviation for the optimum setting. Digital inputs of the DDS IC are then set in order to shift the phase. The programming of the frequency and operation modes of the DDS IC is performed via the SPI interface.

As the output of the phase detector is between 0 and $1.8 \mathrm{~V}$, an external voltage reference is used to maximize the resolution of the internal 10-bit analog digital converter (ADC). Unfortunately the minimum conversion time for each sample is about $100 \mu \mathrm{s}$; it takes about $600 \mu$ s for all phase values to digitize. This is no drawback compared to the analog phase controller which needed a few ms for setting the phase. The phase control cycle is: digitizing the phase signals, computing the phase differences, setting the four control outputs to shift the phase of the DDS outputs.

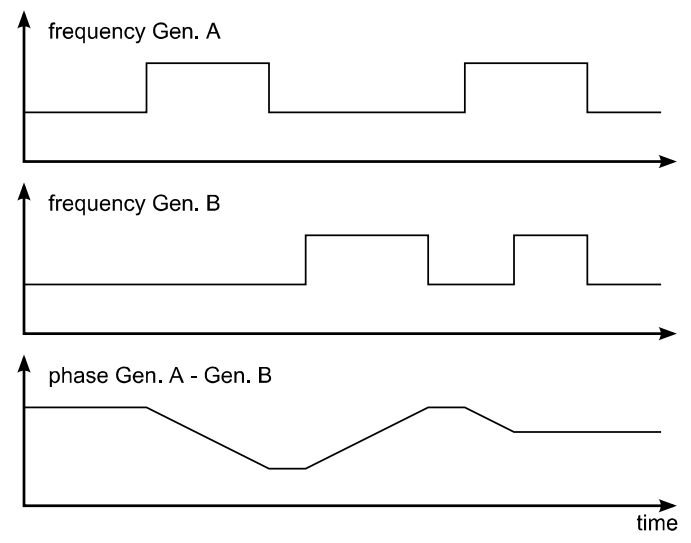

Fig. 5 As the phase is the integral of the frequency difference over time, the phase can also be changed by shifting frequency. If the frequency of Gen. A is raised, the phase difference between Gen. A and Gen. B is decreasing, if the frequency of Gen. B is raised the phase difference is increasing.

\section{Conclusions}

Open hardware devices offer a convenient way to keep old hardware running because one can save time to develop a microcontroller system. The software development environment of the Arduino with a large number of available libraries eases the implementation of standard applications like handling input/output interfaces or controlling displays. The concept of open hardware is at the moment emerging, systems with 16- or even 32-bit processors are getting available, which are powerful enough to cope with complex tasks like fast signal processing [9].

\section{Acknowledgements}

I would like to thank Francesco Monaco from the ECRH group for providing the rotary encoder during the development of the stub tuner position encoder. Johann Kneidl and Gerhard Siegl did a great job designing the mechanical components necessary for the modification of the stub tuners.

\section{References}

[1] O.Gruber, ASDEX Upgrade enhancements in view of ITER application, Fusion Engineering and Design 84 (2009)

[2] A. Kallenbach et al., Recent ASDEX Upgrade Results and Future Extension Plans, IEEE Transactions on Plasma Science, Vol. 40, No. 3, March 2012

[3] K. Behler et al., Real-time diagnostics at ASDEX Upgrade - Architecture and operation, Fusion Engineering and Design 83 (2008), p. 305

[4] V. Bobkov et al., ICRF Operation with Improved Antennas in a Full W-wall ASDEX Upgrade - Status and Developments, 24th IAEA FEC, San Diego, 2012 (to be published)

[5] Arduino Homepage, http://arduino.cc

[6] J. P. Aikio, T. Rahkonen, A Comprehensive Analysis of AM-AM and AM-PM Conversion in an LDMOS RF Power Amplifier, IEEE Transactions on Microwave Theory and Techniques, Vol. 57, No. 2, February 2009

[7] D. Grine et al., Improvement of the phase regulation between two amplifiers feeding the inputs of the $3 \mathrm{~dB}$ combiner in the ASDEX-Upgrade ICRH system, AIP Conf. Proc. 1406, 105 (2011)

[8] K. Polozhiy et al., Influence of the phase shift between antennas on W sputtering in ASDEX Upgrade, 38th EPS Conference on Plasma Physics (2011)

[9] http://arduino.cc/en/Main/ArduinoBoardDue 